XX Міжнародний симпозіум «Методи дискретних особливостей в задачах математичної фізики/Discrete Singularities Methods in Mathematical Physics»,

УДК 517.9

МДОЗМФ/DSMMPh-2021

MSC 45D05

\title{
Dividing of the full reaction of the additional support contacting with the plate into viscous, elastic and inertial components
}

\author{
A.V. Voropay, P.A. Yehorov \\ Kharkov National Automobile and Highway University, Kharkiv, Ukraine \\ E-mail:voropay.alexey@gmail.com
}

\begin{abstract}
An original approach for dividing the reaction of a viscoelastic support into inertial, viscous and elastic components is proposed to assess the effect of various characteristics of additional supports on the deformed state of structural elements. The effectiveness of the proposed approach was tested for a mechanical system consisting of a rectangular isotropic plate of medium thickness, hinged-supported along the contour, and an additional concentrated viscoelastic support, taking into account its mass-inertial characteristics. The deformation of the plate is considered within the framework of Timoshenko's hypotheses. Vibrations of the plate are caused by the applying of an external non-stationary loading. The influence of the additional support is modeled by three independent non-stationary concentrated forces. The paper presents the main analytical relations for obtaining a system of three integral Volterra equations, which is solved numerically and analytically. After performing discretization in time, the system of integral equations is transformed into a system of matrix equations. The resulting system of matrix equations is solved using the generalized Cramer algorithm for block matrices and the Tikhonov regularization method. We point out that the material described is applicable to other objects that have additional supports (beams, plates and shells, which can have different supports along the contour and different shapes in plan). The results of a numerical experiment to determine the components (viscous, elastic and inertial) of the full reaction to the plate, arising due to the presence of an additional support, are presented. The reliability of the proposed approach is confirmed by the coincidence of the results of comparing the reactions found by two methods: numerical-analytical for one complete reaction, as in work [1], and numerical for the full reaction (obtained by adding three components).
\end{abstract}

Key words: medium-thickness plate, identification, nonstationary loading, viscoelastic support, concentrated mass, system of Volterra integral equation, Tikhonov's regularization algorithm.

\section{Распределение полной реакции дополнительной опоры, контактирующей с пластиной, на вязкую, упругую и инерционную составляющие \\ А.В. Воропай, П.А. Егоров \\ Харьковский национальный автомобильно-дорожный университет, Харьков, Украина \\ E-mail:voropay.alexey@gmail.com}

Для оценки влияния на деформированное состояние элементов конструкций различных характеристик дополнительных опор предложен оригинальный подход разделения реакции вязкоупругой опоры на инерционную, вязкую и упругую составляющие. Апробация эффективности предложенного подхода выполнена для механической системы, состоящей из прямоугольной изотропной пластины средней толщины, шарнирно-опёртой по контуру, и дополнительной сосредоточенной вязкоупругой опоры с учётом её массово-инерционной характеристики. Деформирование пластины рассматривается в рамках гипотез Тимошенко. Колебания пла- 


\begin{abstract}
стины обусловлены приложением внешнего нестационарного нагружения. Влияние дополнительной опоры моделируется тремя независимыми нестационарными сосредоточенными силами. В работе приведены основные аналитические соотношения для получения системы трёх интегральных уравнений Вольтерра, которая решается численно-аналитически. После выполнения дискретизации по времени система интегральных уравнений преобразуется в систему матричных уравнений. Полученная система матричных уравнений решается с использованием обобщенного алгоритма Крамера для блочных матриц и метода регуляризации А. Н. Тихонова. Укажем, что изложенный материал применим и для других объектов, имеющих дополнительные опоры (балки, пластины и оболочки, которые могут иметь различное опирание по контуру и разные формы в плане). Приведены результаты численного эксперимента по определению составляющих (вязкой, упругой и инерционной) полной реакции на пластину, возникающей из-за наличия дополнительной опоры. Достоверность предлагаемого подхода подтверждается совпадением результатов сопоставления реакций найденных двумя методами: численно-аналитическим для одной полной реакции, как в работе [1], и численным для суммарной реакции (полученной, сложением трех составляющих).
\end{abstract}

Ключевые слова: пластина средней толщины, идентификация, нестационарное нагружение, вязкоупругая опора, сосредоточенная масса, система интегральных уравнений Вольтерра, регуляризирующий алгоритм А.Н. Тихонова.

\title{
1. Введение и постановка задачи
}

Данная статья является продолжением исследований нестационарного деформирования механических систем, состоящих из балок и пластин, которые, кроме основного опирания по контуру, имеют дополнительные сосредоточенные вязкоупругие опоры. В предыдущих работах $[1,2]$ приведены решения прямых и обратных задач для пластин с дополнительной вязкоупругой опорой. В статье [1] основное внимание посвящено учету массово-инерционной характеристики дополнительной вязкоупругой опоры при моделировании, а в работе [2] предложен метод разделения реакции вязкоупругой опоры на две составляющие (вязкую и упругую). В настоящей работе развивается подход, предложенный в [2] и предполагается получить все три составляющие реакции вязкоупругой опоры с учетом её массово-инерционной характеристики.

Методика определения реакции дополнительной опоры, описанная в работе [1], демонстрирует достаточно высокую эффективность, влияние вязкоупругой опоры с учётом массы, заменялось одной реакцией:

$$
R_{i}(t)=m \frac{d^{2} w(t)}{d t^{2}}+\kappa \frac{d w(t)}{d t}+c w(t),
$$

что позволяло определить ее в результате решения прямой задачи.

Однако для задач, связанных с идентификацией параметров вязкоупругой опоры (жесткости, вязкости и массы), или при оптимальном проектировании дополнительных опор, снижающих амплитуды прогибов, определение полной реакции недостаточно. Необходимо знать отдельно влияние вязкой и упругой, а также инерционной составляющих реакции. В этом случае целесообразно рассмотреть задачу в следующей постановке: пусть прямоугольная пластина имеет две независимые дополнительные опоры - вязкую (демпфирующую) и линейно-упругую, которые приложены в двух разных точках (рис. 1), также к пластине присоединена сосредоточенная масса, вес которой равен весу дополнительной вязкоупругой опоры. Считается, что точка $D$ с координатами проекции в срединной плоскости пластины 
XX Міжнародний симпозіум «Методи дискретних особливостей в задачах математичної фізики/Discrete Singularities Methods in Mathematical Physics»,

\section{МДОЗМФ/DSMМРh-2021}

$\left(x_{D}, y_{D}\right)$ - это место контакта с демпфером, действие которого заменяется реакцией $R_{D}(t)$, точка $C$ с координатами $\left(x_{C}, y_{C}\right)$ - место контакта с линейно-упругой дополнительной опорой (своего рода жесткой пружиной), реакция которой $R_{C}(t)$, а точка $M$ с координатами $\left(x_{M}, y_{M}\right)-$ место присоединения сосредоточенной массы. В общем случае предполагается, что точки $C, D$ и $M$ не совпадают, хотя с точки зрения механики, если точки расположены на небольшом расстоянии, то в силу принципа суперпозиции совместное воздействие вязкой и линейно-упругой опор, а также сосредоточенной массы эквивалентно действию вязкоупругой опоры с учётом её массово-инерционных характеристик:

$$
R(t)=R_{M}(t)+R_{D}(t)+R_{C}(t) .
$$

Такое решение задачи оказалось справедливо и в частном случае, когда точки $C, D$ и $M$ совпадают, поскольку разрешающая система уравнений получилась не вырожденной.

\section{2. Методика решения задачи}

Выражение для прогиба пластины в случае, когда на неё девствует несколько независимых поперечных нагрузок $\left(P_{i}(t), i \in \overline{1, N}\right)$, имеет следующий вид [3]:

$$
w(x, y, t)=\sum_{i=1}^{N} \int_{0}^{t} K_{i}(x, y, t-\tau) \cdot P_{i}(\tau) d \tau,
$$

где $w(x, y, t)$ - изменение прогиба пластины во времени; $K_{i}(x, y, t)-$ конечноразностное ядро интеграла свертки, для $i$-той нагрузки в точке пластины с координатами $(x, y)$. Если считать, что возмущающая сила, а также реакции дополнительных опор и массы являются сосредоточенными нагрузками, то соответствующие ядра $K_{i j}(t)$ в интегралах сверток для шарнирно-опертой пластины средней толщины типа С. П. Тимошенко будут иметь вид:

$$
\begin{aligned}
& K_{i j}(t)=\sum_{k=1}^{\infty} \sum_{n=1}^{\infty}\left[\Omega_{1 k n} \cdot \sin \omega_{1 k n}(t)-\Omega_{2 k n} \cdot \sin \omega_{2 k n}(t)\right] \cdot \sin \left(\frac{k \pi x_{i}}{l_{1}}\right) \cdot \sin \left(\frac{n \pi y_{i}}{l_{2}}\right) \times \\
& \times \sin \left(\frac{k \pi x_{j}}{l_{1}}\right) \cdot \sin \left(\frac{n \pi y_{j}}{l_{2}}\right),
\end{aligned}
$$

где $\omega_{1 k n}$ и $\omega_{2 k n}-$ собственные частоты колебаний пластины; $l_{1}$ и $l_{2}$ - длина и ширина пластины; $\left(x_{i}, y_{i}\right)$ - координаты точки, в которой прикладывается $i$-тая нагрузка, а $\left(x_{j}, y_{j}\right)$ - это координаты $j$-той точки, в которой определяется изменение прогиба во времени.

Укажем, что реакции $R_{C}(t), R_{D}(t)$ и $R_{M}(t)$, действующие со стороны дополнительных опор и массы на пластину, считались условно отрицательными (взяты в выражениях (2) со знаком минус), действия со стороны пластины на массу, демпфер и «пружину» считались равными по величине, но условно положительными (для удобства записи):

$$
R_{M}(t)=m \frac{d^{2} w\left(x_{M}, y_{M}, t\right)}{d t^{2}}, R_{D}(t)=\kappa \cdot \frac{d w\left(x_{D}, y_{D}, t\right)}{d t}, R_{C}(t)=c \cdot w\left(x_{C}, y_{C}, t\right) .
$$


Из соотношений (4) для прогибов в точках $\left(x_{M}, y_{M}\right),\left(x_{D}, y_{D}\right)$ и $\left(x_{C}, y_{C}\right)$ можно получить следующие выражения:

$$
w_{M}(t)=\frac{1}{m} \int_{0}^{t} R(\tau)(t-\tau) d \tau, w_{D}(t)=\frac{1}{\kappa} \int_{0}^{t} R_{D}(\tau) d \tau \text { и } w_{C}(t)=\frac{1}{c} R_{C}(t) .
$$

Приравняем соответствующие пары выражений (2) и (5) для точек контакта с демпфером $\left(x_{D}, y_{D}\right)$, линейно-упругой дополнительной опорой $\left(x_{C}, y_{C}\right)$ и сосредоточенной массой $\left(x_{M}, y_{M}\right)$ получим 3 интегральных соотношения. В случае, когда $P(t)$ известна, эти выражения будут являться системой интегральных уравнений относительно неизвестных сил $R_{C}(t), R_{D}(t)$ и $R_{M}(t)$. После переноса известных слагаемые в правую часть получим:

$$
\left\{\begin{array}{l}
\int_{0}^{t} K_{C C}(t-\tau) \cdot R_{C}(\tau) d \tau+\frac{R_{C}(t)}{c}+\int_{0}^{t} K_{C D}(t-\tau) \cdot R_{D}(\tau) d \tau+ \\
+\int_{0}^{t} K_{C M}(t-\tau) \cdot R_{M}(\tau) d \tau=\int_{0}^{t} K_{P C}(t-\tau) \cdot P(\tau) d \tau ; \\
\int_{0}^{t} K_{D C}(t-\tau) \cdot R_{C}(\tau) d \tau+\int_{0}^{t} K_{D D}(t-\tau) \cdot R_{D}(\tau) d \tau+ \\
+\int_{0}^{t} \frac{R_{D}(\tau)}{\kappa} d \tau+\int_{0}^{t} K_{D M}(t-\tau) \cdot R_{M}(\tau) d \tau=\int_{0}^{t} K_{P D}(t-\tau) \cdot P(\tau) d \tau ; \\
\int_{0}^{t} K_{M C}(t-\tau) \cdot R_{C}(\tau) d \tau+\int_{0}^{t} K_{M D}(t-\tau) \cdot R_{D}(\tau) d \tau+ \\
+\int_{0}^{t} K_{M M}(t-\tau) \cdot R_{M}(\tau) d \tau+\frac{1}{m} \int_{0}^{t} R(\tau)(t-\tau) d \tau=\int_{0}^{t} K_{P M}(t-\tau) \cdot P(\tau) d \tau,
\end{array}\right.
$$

где $P(t)$ - внешняя возмущающая нестационарная нагрузка, $K_{i j}(t)$ - ядра, записанные для конкретной $i$-той нагрузки в $j$-той точке, вид которых будет приведен ниже, $R_{C}(t), R_{D}(t)$ и $R_{M}(t)$ - соответствующие реакции в точках контакта с массой, демпфером и линейно-упругой опорой.

Для решения системы интегральных уравнений (6) была выполнена дискретизация по времени ( $\Delta t=T / J$, где $T$ - исследуемый промежуток, а $J$ - число шагов дискретизации) методом частичного интегрирования. То есть на малых дискретных участках времени сила $P(t)=P(j \cdot \Delta t)=P_{j}$, а также реакции $R_{C}(j \cdot \Delta t)$, $R_{D}(j \cdot \Delta t)$ и $R_{M}(j \cdot \Delta t)$ считались условно постоянными. В результате получена следующая система матричных уравнений:

$$
\left\{\begin{array}{l}
\mathbf{A}_{C C} \cdot \mathbf{R}_{C}+\mathbf{A}_{C D} \cdot \mathbf{R}_{D}+\mathbf{A}_{C M} \cdot \mathbf{R}_{M}=\mathbf{A}_{P C} \cdot \mathbf{P} \\
\mathbf{A}_{D C} \cdot \mathbf{R}_{C}+\mathbf{A}_{D D} \cdot \mathbf{R}_{D}+\mathbf{A}_{D M} \cdot \mathbf{R}_{M}=\mathbf{A}_{P D} \cdot \mathbf{P} \\
\mathbf{A}_{M C} \cdot \mathbf{R}_{C}+\mathbf{A}_{M D} \cdot \mathbf{R}_{D}+\mathbf{A}_{M M} \cdot \mathbf{R}_{M}=\mathbf{A}_{P M} \cdot \mathbf{P}
\end{array}\right.
$$


XX Міжнародний симпозіум «Методи дискретних особливостей в задачах математичної фізики/Discrete Singularities Methods in Mathematical Physics»,

\section{МДОЗМФ/DSMМPh-2021}

где матрицы $\mathbf{A}_{P C}, \mathbf{A}_{P D}, \quad \mathbf{A}_{P M}$ соответствуют ядрам $K_{P C}(t-\tau), \quad K_{P D}(t-\tau)$ и $K_{P M}(t-\tau)$.

Укажем, что систему матричных уравнений (7) можно представить в виде блочной матрицы. Матрицы $\mathbf{A}_{i j}$ соответствуют частично проинтегрированным ядрам $K_{i j}(t-\tau)$ при $i \neq j$. В случае, когда $i \neq j$ матрицы $\mathbf{A}_{i i}$, находящиеся на главной диагонали блочной матрицы модифицируются с учетом особенности (для дополнительной упругой и вязкой опоры, а также сосредоточенной массы).

Матрица $\mathbf{A}_{C C}$ эквивалентна выражению $\mathbf{A}_{C C}=\mathbf{A}_{C C}^{*}+c^{-1} \cdot \mathbf{E}$, где матрица $\mathbf{A}_{C C}^{*}$ соответствует частично проинтегрированному ядру $K_{C C}(t-\tau)$, а $\mathbf{E}$ - единичная матрица. Матрицы $\mathbf{A}_{D D} \quad$ и $\mathbf{A}_{M M}$ соответствуют интегралам $\int_{0}^{t}\left[K_{D D}(t-\tau)+\frac{1}{\kappa}\right] \cdot R_{D}(\tau) d \tau$ и $\int_{0}^{t}\left[K_{D D}(t-\tau)+\frac{t-\tau}{m}\right] \cdot R_{M}(\tau) d \tau$.

Система (8) решается с использованием обобщенного алгоритма Крамера для блочных матриц [4] и регуляризирующего алгоритма А. Н. Тихонова [5]. В результате решения (8) определяются $\mathbf{R}_{C}, \mathbf{R}_{D}$ и $\mathbf{R}_{M}$, являющиеся дискретными аналогами неизвестных независимых реакций $R_{C}(t), R_{D}(t)$ и $R_{M}(t)$.

\section{3. Численный эксперимент}

При расчетах срединная плоскость пластины была связана с плоскостью $x O y$ декартовой системы координат. Расчеты производились при следующих значениях параметров: $\rho=7890$ кг $/ \mathrm{m}^{3} ; v=0.3 ; E=2.07 \cdot 10^{11}$ Па; $h=0.04 \mathrm{м} ; l_{l}=0.6 \mathrm{м}, l_{2}=0.4$ м. Коэффициент жесткости дополнительной опоры $c=10^{8} \mathrm{H} / \mathrm{M}$, коэффициент линейновязкого демпфирования $\kappa=4 \cdot 10^{4} \mathrm{H} /($ м/с); величина массы опоры $m=5$ кг. Число членов в соответствующих двойных рядах Фурье $50 \times 50$. Координаты точки приложения возмущающей нагрузки имеют значения: $x_{0}=0.4 \mathrm{~m}, y_{0}=0.2 \mathrm{~m}$; координаты точки крепления дополнительной вязкоупругой опоры к пластине: $x_{\mathrm{CD}}=0.2 \mathrm{M}$, $y_{\mathrm{CD}}=0.2 \mathrm{м}$; координаты точки, в которой исследовалось изменение прогиба пластины: $x_{\mathrm{S}}=0.3 \mathrm{M}, y_{\mathrm{S}}=0.2 \mathrm{M}$.

Составляющие реакции между пластиной и дополнительной вязкоупругой опорой, определенные в результате решения численно-аналитического решения системы интегральных уравнений (6), приведены на рис. 2: 1-я кривая - суммарная реакции вязкоупругой опоры $R_{\Sigma}(t)=R_{C}(t)+R_{D}(t)+R_{M}(t) ; 2$-я кривая - упругая составляющая $R_{C}(t) ; 3$-я кривая - вязкая составляющая $R_{D}(t) ; 4$-я кривая - инерционная составляющая $R_{M}(t)$.

На рис. 3 показаны кривые: $R_{\Sigma}(t)$ - суммарная реакция, которая также приведена выше (рис. 2), полученная численно, а $R_{f}(t)$ - это реакция вязкоупругой опоры (1), вычисленная согласно методике описанной в работе [1] (как одна полная реакция).

Укажем, что незначительное расхождение кривых $R_{\Sigma}(t)$ и $R_{f}(t)$ можно объяснить различием используемых методов, а именно: кривая $R_{f}(t)$ полученная чис- 
ленно-аналитическим методом, описанным в работе [1], является более точной, а суммарная реакция $R_{\Sigma}(t)$, полученная сложением трёх составляющих, является некоторым усреднением, и поэтому кажется сглаженной. Близость кривых указанных кривых доказывает правильность решения и возможность использования любого из двух подходов для определения реакции вязкоупругой опоры с учётом её массово-инерцонных характеристик.

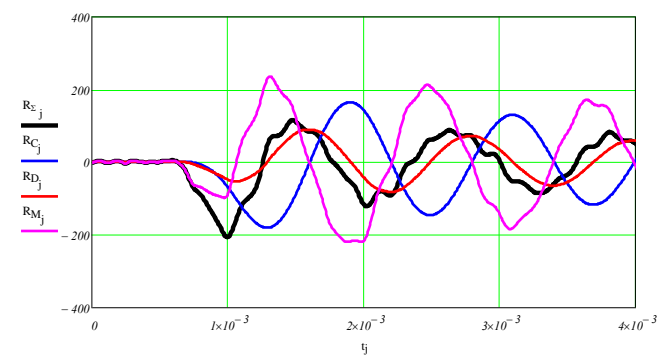

Рис. 2 - Составляющие реакции вязкоупругой опоры $R_{C}(t), R_{D}(t), R_{M}(t)$ и их суммарное воздействие $R_{\Sigma}(t)$.

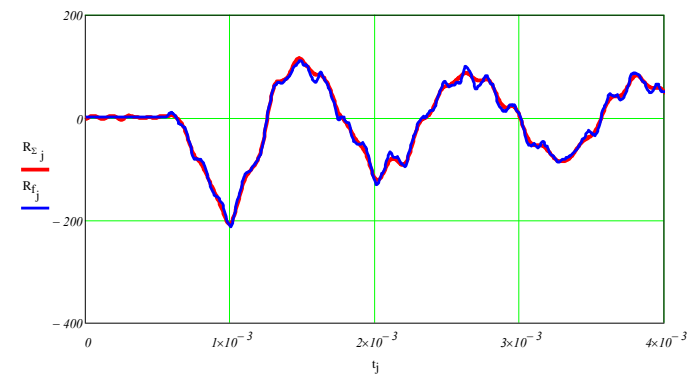

Рис. 3 - Реакции вязкоупругой опоры $R_{\Sigma}(t)$ и $R_{f}(t)$, найденные двумя разными методами.

\section{4. Выводы}

В работе описан оригинальный подход, при котором воздействие дополнительной вязкоупругой опоры на пластину моделируется в виде трёх неизвестных независимых нестационарных сил - упругой, вязкой и инерционной составляющих реакции между пластиной и дополнительной опорой. Достоверность предлагаемого подхода подтверждается путем совпадения результатов сопоставления реакций найденных двумя методами: численно-аналитически для одной полной реакции, как в работе [1], и численно - суммарной (сложением трех составляющих).

\section{ЛИТЕРАТУРА}

1. Воропай А.В., Егоров П.А. Учёт влияния массово-инерционной характеристики дополнительной вязкоупругой опоры при нестационарном деформировании прямоугольной пластины. Вісник НТУ "ХПI". Сер.: Математичне моделювання в техніці та технологіях. 2020. № 1. С. 15-23.

2. Воропай А.В. Распределение вязкой и упругой составляющих в реакции дополнительной вязкоупругой опоры, контактирующей с пластиной. Вісник НТУ «ХПІ». Серія: Математичне моделювання в технічі та технологіях. 2016. №16 (1188). C. 16-22.

3. Янютин Е.Г., Воропай А.В., Поваляев С.И., Янчевский И.В. Идентификация нагрузок при импульсном деформировании тел. Монография в 2-х частях. Часть ІІ. Харьков: Изд-во ХНАДУ, 2010. 212 с.

4. Гантмахер Ф.Р. Теория матриц. М.: Наука, 1967. 576 с.

5. Тихонов А.Н., Гончаровский А.В. и др. Регуляризирующие алгоритмы и априорная информация. М.: Наука, 1983. 200 с. 
XX Міжнародний симпозіум «Методи дискретних особливостей в задачах математичної фізики/Discrete Singularities Methods in Mathematical Physics»,

\section{МДОЗМФ/DSMМPh-2021}

\section{Розподіл повної реакції додаткової опори, що контактує 3 пластиною, на в'язку, пружну та інерційну складові \\ О.В. Воропай, П.А. Єгоров}

Харківський національний автомобільно-дорожній університет, Харків, Україна E-mail:voropay.alexey@gmail.com

3 метою оцінки впливу на деформований стан елементів конструкцій різних характеристик додаткових опор запропоновано оригінальний підхід поділу реакції в'язкопружної опори на інерційну, в'язку та пружну складові. Апробація ефективності запропонованого підходу виконана для механічної системи, що складається 3 прямокутної ізотропної пластини середньої товщини, шарнірно-обпертої уздовж контуру, і додаткової зосередженої вязкопружної опори з урахуванням іiі масовоінерційної характеристики. Деформування пластини розглядається в рамках гіпотез Тимошенко. Коливання пластини обумовлені прикладенням зовнішнього нестаціонарного навантаження. Вплив додаткової опори моделюється трьома незалежними нестаціонарними зосередженими силами. В роботі наведено основні аналітичні співвідношення для отримання системи трьох інтегральних рівнянь Вольтерра, яка розв'язується чисельно-аналітично. Після виконання дискретизації за часом система інтегральних рівнянь перетворюється в систему матричних рівнянь. Отримана система матричних рівнянь розв'язується 3 використанням узагальненого алгоритму Крамера для блокових матриць і методу регуляризації А. М. Тихонова. Зазначимо, що викладений матеріал може бути застосований і для інших об'єктів, що мають додаткові опори (балки, пластини і оболонки, які можуть мати різне спирання по контуру і різні форми в плані). Приведено результати чисельного експерименту по визначенню складових (в'язкою, пружною і інерційної) повної реакції на пластину, що виникає через наявність додаткової опори. Достовірність запропонованого підходу під-підтверджується збігом результатів зіставлення реакцій знайдених двома методами: чисельно-аналітичного для однієї повної реакції, як у роботі [1], i чисельного для сумарної реакції (отриманої, складанням трьох складових).

Ключові слова: пластина середньої товщини, ідентифікація, нестаціонарне навантаження, в'язкопружна опора, зосереджена маса, система інтегральних рівнянь Вольтерра, регуляризуючий алгоритм А.М. Тихонова. 